\title{
Limitations of the ACS NSQIP in Thyroid Surgery
}

\author{
Rebecca S. Sippel, MD and Herbert Chen, MD \\ Section of Endocrine Surgery, Department of Surgery, University of Wisconsin, Madison, WI
}

During the early $1990 \mathrm{~s}$, the Department of Veterans Affairs first began to develop a risk-adjusted outcomes program, which in 1994 became the VA National Surgical Quality Improvement Program (NSQIP). Due to the significant success of this program in the VA system for decreasing both morbidity and mortality, there was significant interest in expanding this program outside of the VA system. In 2001, the American College of Surgeons (ACS) began a pilot program in collaboration with the VA. This program has expanded rapidly and is now considered the best available tool for surgical quality improvement. ${ }^{1}$

Designed as a tool to help improve surgical outcomes at individual hospitals, the participant use data file, which is available free to all participating hospitals, has become a popular data source for outcomes researchers in surgery. The ACS NSQIP is a powerful database, because it is now collecting detailed outcome data for nearly 300,000 patients per year. They collect data on preoperative risk factors, intraoperative variables, and 30-day morbidity and mortality. Although these data are incredibly useful to review the outcomes of high-risk procedures, such as vascular surgery, esophagectomy, and colon surgery, the applicability to lower risk operations, such as thyroidectomy, can be questioned.

The outcomes that are relevant to thyroidectomy are bleeding that requires a reoperation, recurrent laryngeal nerve paresis (transient or permanent), and hypoparathyroidism (transient or permanent). Unfortunately in the currently available form of the ACS NSQIP, none of these procedure-specific complications are tracked. Without examining these procedure-specific complications, it is challenging to make any conclusions regarding the outcomes of thyroidectomy using this data set. This is a

(C) Society of Surgical Oncology 2011

Published Online: 14 July 2011

R. S. Sippel, MD

e-mail: sippel@surgery.wisc.edu powerful dataset due to its size, but one must be careful when interpreting the data that the outcomes you are reporting actually represent what you think they do.

Goldfarb et al. have provided an important study that focuses on the outcomes of thyroidectomy for benign and malignant disease. ${ }^{2}$ They have shown that the mean operative time for malignancy is longer by $4 \mathrm{~min}$ and the average length of stay is longer by 0.04 days. They also have shown that the rate of return to the operating room (OR) was higher in the malignant group (3.8 vs. 1.8\%) and that overall complications, including return to the OR, was higher (4.9 vs. 3.3\%), although complications excluding a return to the OR were not different. Their data clearly show these differences and the statistical calculations confirm that the differences are significant. However, there are some clinical differences that exist between these two groups that are not controlled for within the NSQIP dataset that could potentially explain all of these findings.

Patients with a known malignancy will undergo a total thyroidectomy in most cases and often a lymph node dissection. Lobectomies would typically only be performed for benign or indeterminant disease. Lobectomies are shorter operations and often are performed as an outpatient with a shorter length of stay (LOS). The difference in operative time and LOS may be due to the fact that the benign group underwent "less surgery."

Return to the OR is a variable tracked by ACS NSQIP, and it records any return to the operating room within 30 days. Because bleeding that requires a return to the OR is a clinically significant variable in thyroid surgery, one would like to assume that these two variables are synonymous. Unfortunately, we know that patients may return to the OR after thyroidectomy, not only for bleeding or infection, but also for additional surgery if cancer is diagnosed postoperatively. Whereas some surgeons will wait several months to do a completion thyroidectomy, many surgeons will opt to do it as soon as the cancer diagnosis is made (within 30 days). These planned 
completion thyroidectomies will be coded as a return to the OR within 30 days, which according to NSQIP is a complication. In another paper that we previously published that examined bleeding and deep venous thrombosis risk after thyroidectomy, we controlled for this variable by excluding all patients with an initial lobectomy who had a final diagnosis of cancer, assuming that a return to the OR in this cohort might not actually be a complication but a planned returned. ${ }^{3}$ Once we excluded these patients, our rate of return to the OR for reasons other than infection or completion thyroidectomy was only $1.58 \%$, significantly lower than the rate of $2.2 \%$ in this study. Supporting this finding is the fact that Goldfarb's analysis shows that total thyroidectomy was associated with a decreased risk in returning to the OR, suggesting that a significant number of the returns to the OR were not complications but planned returns as part of appropriate clinical care. Although there is a difference in the rate of return to OR, one must be cautious in the interpretation of this finding, because there may be other clinical variables at play that are not fully accounted for in the NSQIP dataset or in this analysis.

Therefore, the endocrine surgery group at Massachusetts General Hospital has highlighted an important issue in thyroid surgery with the currently available, limited dataset. The ACS NSQIP has realized that to make the data helpful to endocrine and other specialty surgeons, it is important to have disease-specific outcome measures. Thyroidectomy has been recognized as one of those procedures in which the current dataset has significant limitations. As of this year, institutions now have the opportunity to participate in a thyroid-specific dataset, which will collect variables and complication data specific to thyroid surgery. This new enhanced dataset will have much greater utility for thyroid surgeons and will hopefully allow us to really address what influences outcomes in thyroid surgery and how we can learn to do it better in the future.

\section{REFERENCES}

1. Hall BL, Hamilton BH, Richards K, Bilimoria KY, Cohen ME, Ko CY. Does surgical quality improve in the American College of Surgeons National Surgical Quality Improvement Program? An evaluation of all participating hospitals. Ann Surg. 2009; 250(3):363-76.

2. Goldfarb M, Perry Z, Hodin R, Parangi S. Medical and surgical risks in thyroid surgery: lessons from the NSQIP. Ann Surg. doi: 10.1245/s10434-011-1938-2.

3. Roy M, Rajamanickam V, Chen H, Sippel R. Is DVT prophylaxis necessary for thyroidectomy and parathyroidectomy? Surgery. 2010;148(6):1163-8; discussion 8-9. 